\title{
Calcinose na Infância, um Desafio Terapêutico
}

\section{Calcinosis in Juvenile Dermatomyositis, A Therapeutic Challenge}

\author{
Tânia Caroline Monteiro de Castro( ${ }^{(1)}$, Edson Yamashita $^{(2)}$, Maria Teresa R. A. Terreri ${ }^{(3)}$, \\ Claúdio Arnaldo Len ${ }^{(4)}$, Maria Odete Esteves Hilário ${ }^{(5)}$
}

\section{RESUMO}

A calcinose é uma complicação comum e incapacitante da dermatomiosite. Ela constitui uma característica marcante da doença, ocorrendo principalmente em pacientes pediátricos. Pouco se sabe a respeito de sua fisiopatologia e não existe um tratamento reconhecido universalmente. Este trabalho relata dois casos de dermatomiosite juvenil e calcinose grave com melhora clínica com o uso do alendronato e do diltiazen em um paciente e da imunoglobulina endovenosa em outro.

Palavras-chave: dermatomiosite juvenil, calcinose, alendronato, diltiazen, imunoglobulina.

\section{INTRODUÇÃO}

A calcinose ou calcificação de tecidos não-articulares é uma complicação freqüente da dermatomiosite juvenil $(\mathrm{DMJ})$, com incidência entre $10 \%$ e $70 \%^{(1,2)}$. Pode ser dolorosa e muito debilitante, resultando, às vezes, em incapacidade funcional. A patogênese é desconhecida, mas parece envolver a ação de células inflamatórias, citocinas e proteínas da matriz mineralizada, como osteocalcina, entre outras $^{(3)}$. Atraso no início do tratamento, vasculopatia e doença grave são fatores de risco para o aparecimento de calcinose nesses pacientes ${ }^{(4-6)}$.

Muitas medicações têm sido utilizadas para tratar calcinose, algumas com sucesso em poucos relatos de caso. Entre elas destacam-se: hidroxicloroquina, imunoglobulina endovenosa, ciclosporina, colchicina, probenecid, warfarin, diltiazen, hidróxido de alumínio, triamcinolona tópica, infliximabe e alendronato. Os resultados dessas terapias têm sido contraditórios, e benefícios consistentes não têm sido encontrados ${ }^{(7-11)}$.

\begin{abstract}
Calcinosis is a common and debilitating complication of dermatomyositis. It is a hallmark of the disease, occurring mainly in pediatric patients. Little is known about its pathophysiology, and there is no universally recognized treatment. This is a report of two children with juvenile dermatomyositis and severe calcinosis who showed improvement with the use of alendronate and diltiazen in one patient, and intravenous immunoglobulin in the other.
\end{abstract}

Keywords: juvenile dermatomyositis, calcinosis, alendronate, diltiazen, immunoglobulin.

Os autores relatam e discutem o uso de algumas dessas medicações, como o alendronato, o diltiazen e a imunoglobulina endovenosa (IGEV) no tratamento da calcinose em dois pacientes com DMJ e fazem uma revisão da literatura das medicações mais utilizadas nesta que é a seqüela mais característica e a menos compreendida da DMJ.

\section{RELATO DE CASO}

\section{CASO 1}

Paciente de 10 anos de idade, sexo feminino, teve diagnóstico de DMJ aos 4 anos e 10 meses de idade, após um ano com história de fraqueza muscular e lesões cutâneas com fotossensibilidade. Na época, relatava dificuldade para subir escadas, segurar objetos, claudicação, quedas da própria altura e artrite em joelho esquerdo, sem outras queixas clínicas.

\footnotetext{
Recebido em 29/09/06. Aprovado, após revisão, em 16/11/06.

1. Médica Assistente do Setor de Reumatologia Pediátrica da Disciplina de Alergia, Imunologia Clínica e Reumatologia do Departamento de Pediatria da Unifesp-EPM.

2. Médico Residente do Setor de Reumatologia Pediátrica da Disciplina de Alergia, Imunologia Clínica e Reumatologia do Departamento de Pediatria da Unifesp-EPM.

3. Professora Afiliada do Setor de Reumatologia Pediátrica da Disciplina de Alergia, Imunologia Clínica e Reumatologia do Departamento de Pediatria da Unifesp-EPM.

4. Professor Adjunto do Setor de Reumatologia Pediátrica da Disciplina de Alergia, Imunologia Clínica e Reumatologia do Departamento de Pediatria da Unifesp-EPM.

5. Professora Associada e Chefe do Setor de Reumatologia Pediátrica da Disciplina de Alergia, Imunologia Clínica e Reumatologia do Departamento de Pediatria da UNIFESP-EPM

Endereço para correspondência: Tânia Caroline Monteiro de Castro, Rua dos Otonis, 725, CEP 04025-002, São Paulo, SP, Brasil, e-mail: taniacaroline@uol.com.br.
} 
Ao exame físico, apresentava edema eritematovioláceo peripalpebral (heliotropo), pápulas avermelhadas em metacarpofalangeanas e interfalangeanas (pápulas de Gottron), flexo de cotovelos e joelhos, e vasculite de mãos e pés. Não foi realizado escore de força muscular, pois não houve colaboração da paciente.

Os exames laboratoriais mostraram aumento de enzimas musculares com creatininoquinase $(\mathrm{CPK})$ de $3307 \mathrm{U} / \mathrm{L}$ (normal de 38 a $174 \mathrm{U} / \mathrm{L}$ ), aldolase de $13 \mathrm{U} / \mathrm{L}$ (normal até 7,6 U/L), desidrogenase lática (DHL) de $862 \mathrm{U} / \mathrm{L}$ (normal de 240 a $480 \mathrm{U} / \mathrm{L}$ ), transaminase glutâmicooxalacética (TGO) de $40 \mathrm{U} / \mathrm{L}$ (normal até $38 \mathrm{U} / \mathrm{L}$ ) e velocidade de hemossedimentação (VHS) de $5 \mathrm{~mm}$ na primeira hora. Apresentava anticorpo antinuclear (AAN) positivo com padrão pontilhado fino $\geq 1: 640$, anti-DNA e anti-ENA negativos, hemograma e urina I normais. A biópsia muscular mostrou inflamação com infiltrado linfoplasmocitário, intersticial e perivascular. A capilaroscopia periungueal mostrou padrão escleroderma (SD), com intensa ectasia e grau elevado de deleção capilar.

Iniciadas pulsoterapia endovenosa com metilprednisolona $30 \mathrm{mg} / \mathrm{kg} /$ dia por três dias e prednisona oral $1 \mathrm{mg} / \mathrm{kg} /$ dia, além de protetor solar. Após seis meses de tratamento, foi introduzido metotrexate (MTX) e aumentado progressivamente até $0,8 \mathrm{mg} / \mathrm{kg} / \mathrm{semana}$ por via subcutânea, além de ácido fólico. Após dois anos do início do quadro clínico e um ano de tratamento, com pouca aderência, a paciente iniciou lesões de calcinose em face anterior da tíbia esquerda e face posterior da tíbia direita. Introduzido alendronato $35 \mathrm{mg} /$ semana e claritromicina por sete dias (devido à infecção secundária após drenagem espontânea das lesões). Após seis meses com alendronato, surgiram lesões em coxas e região dorsal que apresentaram infecção secundária com necessidade de debridamento (Figura 1). Foi, então, associado diltiazen $4,8 \mathrm{mg} / \mathrm{kg} /$ dia. Nessa ocasião, a paciente não referia fraqueza muscular, as enzimas musculares estavam normais e a densitometria óssea Ll-L4, normal (Z-escore de -1,0 DP).

Após $\mathrm{l}$ ano de uso regular do alendronato e seis meses de uso de diltiazen, houve melhora importante da calcinose (Figura 2). Há dez meses, está assintomática, sem atividade da doença e sem calcinose.

\section{CASO 2}

Paciente de 13 anos de idade, sexo masculino, apresentou aos 2 anos de idade lesões atróficas em couro cabeludo. Três anos após o início do quadro cutâneo, foi

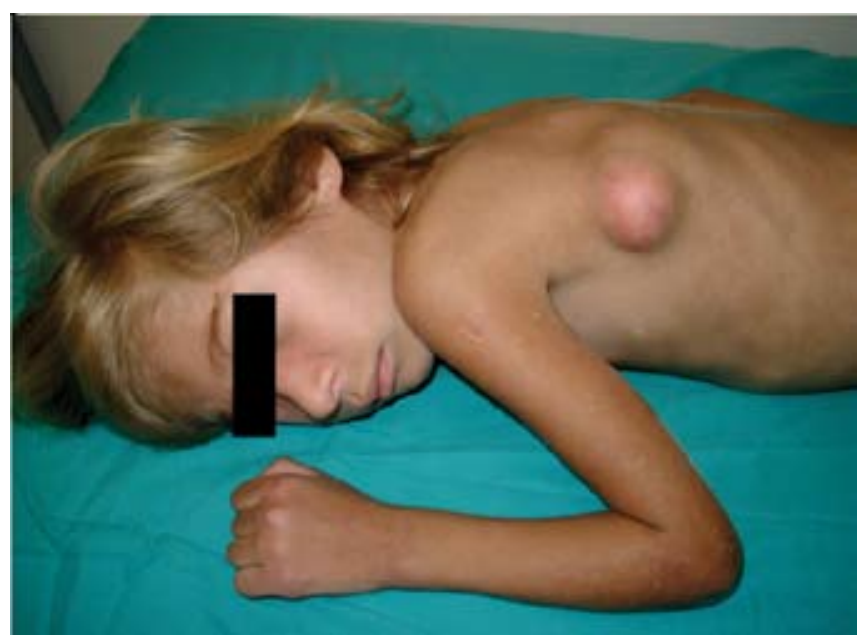

Figura 1 - Calcinose em região dorsal.

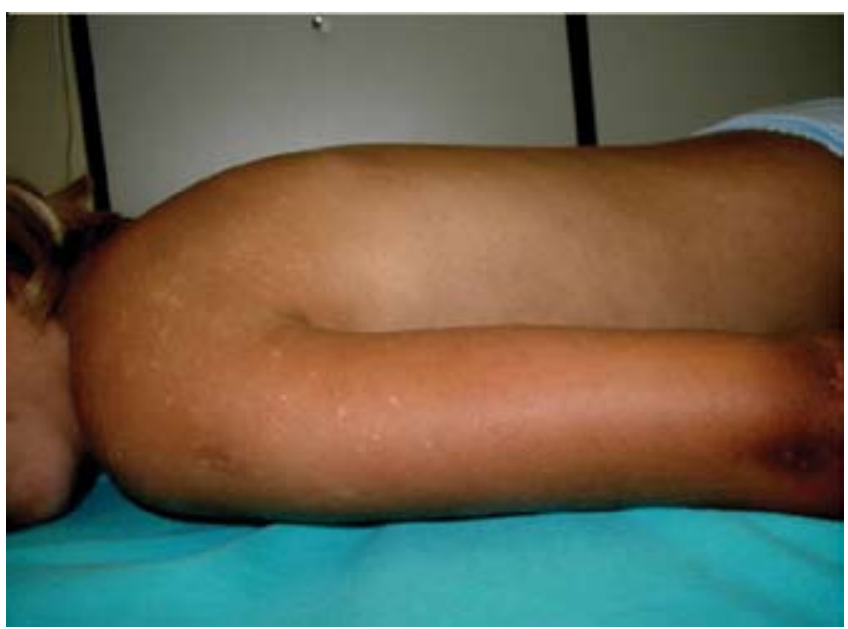

Figura 2 - Resolução total da calcinose.

realizada biópsia de pele, compatível com esclerodermia. Nessa época, iniciou heliotropo, Gottron e pequenas calcificações em superfícies flexoras dos membros superiores, tendo sido acompanhado na dermatologia com hipótese de fibrodisplasia ossificante progressiva (FOP). Como evoluiu com quadro de fraqueza muscular proximal (dificuldade de deambular e de subir escadas), foi realizada biópsia muscular que confirmou o diagnóstico de DMJ.

Ao exame físico, apresentava edema eritematovioláceo peripalpebral (heliotropo), pápulas avermelhadas em metacarpofalangeanas e interfalangeanas (pápulas de Gottron), limitação à extensão e flexão de punhos, flexo de cotovelo direito, limitação à rotação interna e externa de quadris, calcinose em face flexora dos membros superiores e diminuição de força muscular. Os exames solicitados mostraram aumento de enzimas musculares com CPK de $196 \mathrm{U} / \mathrm{L}$, 
aldolase de 11,7 U/L, DHL de $603 \mathrm{U} / \mathrm{L}$, TGO de 57 $\mathrm{U} / \mathrm{L}$ e VHS de $10 \mathrm{~mm}$ na primeira hora. Apresentava hemograma e urina 1 normais, AAN, anti-DNA e anti-ENA negativos, eletroneuromiografia com padrão miopático e cinevideodeglutograma normal. A capilaroscopia periungueal apresentou padrão SD com intensa ectasia e grau elevado de deleção capilar.

Iniciados prednisona oral $1 \mathrm{mg} / \mathrm{kg} / \mathrm{dia}, \mathrm{MTX}$ $10 \mathrm{mg} / \mathrm{semana}(0,4 \mathrm{mg} / \mathrm{kg} / \mathrm{semana})$, posteriormente aumentado para $12,5 \mathrm{mg} / \mathrm{semana}(0,5 \mathrm{mg} / \mathrm{kg} / \mathrm{semana})$, ácido fólico e protetor solar. Após um ano de tratamento, houve aparecimento de calcinose em região extensora de cotovelos e axila direita. Foi introduzido diltiazen $45 \mathrm{mg} /$ dia $(1,8 \mathrm{mg} / \mathrm{kg} /$ dia $)$ e aumentado para $60 \mathrm{mg} /$ dia $(2,4 \mathrm{mg} / \mathrm{kg} /$ dia $)$ e, posteriormente, para $90 \mathrm{mg} /$ dia $(3,2 \mathrm{mg} / \mathrm{kg} / \mathrm{dia})$, após aparecimento de calcinose em região inguinal. Durante um ano e três meses de tratamento com diltiazen, não houve melhora da calcinose. Nessa época, o paciente estava sem atividade clínica ou laboratorial da doença e foram gradativamente suspensas todas as medicações. Dois anos e três meses depois, o paciente apresentou atividade laboratorial (aumento de enzimas musculares e VHS) e aparecimento de calcinose em região palmar direita. Foi reintroduzido diltiazen $90 \mathrm{mg} / \mathrm{dia}(2,3 \mathrm{mg} / \mathrm{kg} / \mathrm{dia})$ por um ano. Como não houve melhora clínica, foi suspenso diltiazen e iniciado alendronato $70 \mathrm{mg} / \mathrm{semana}$. Nos cinco meses seguintes, houve aparecimento de novas lesões de calcinose, foi introduzida IGEV l g/ $\mathrm{kg} /$ dia e suspenso o alendronato após 11 meses de tratamento. A IGEV foi mantida por sete meses, tendo havido melhora da calcinose com desaparecimento de algumas lesões a partir da quinta infusão e redução significativa na extensão da calcinose. Há sete meses, está sem atividade clínica da doença e sem calcinose.

\section{DISCUSSÃO}

A DMJ é uma doença sistêmica, caracterizada por inflamação não-supurativa da musculatura estriada e da pele. A complicação mais comum da DMJ é a calcinose ${ }^{(12)}$. Esta costuma aparecer durante a fase de atividade da doença e, geralmente, está relacionada à doença cutânea grave, à vasculopatia importante, ao curso crônico e ao atraso no diagnóstico e tratamento ${ }^{(4)}$. O aparecimento da calcinose ocorre mais freqüentemente um a três anos após o início da doença, mas tem sido relatada até 20 anos após a instalação desta $^{(13)}$. Os depósitos de cálcio podem aparecer no tecido subcutâneo, na fáscia muscular ou intramuscular, com predileção para áreas propensas a traumas repetidos como cotovelos, joelhos, nádegas e superfícies articulares flexoras, muitas vezes impedindo os movimentos ${ }^{(12,14)}$. Calcificação superficial generalizada formando um exoesqueleto também já foi descrita e parece não estar relacionada ao retardo no diagnóstico e no tratamento como nas demais formas ${ }^{(2)}$. As calcinoses podem ainda causar atrofia muscular secundária, contraturas articulares e úlceras cutâneas com episódios recorrentes de inflamação ou infecção local e extrusão de material cálcico e purulento da lesão ${ }^{(2)}$. Estudo do material da calcinose mostrou presença de macrófagos, interleucinas $1 \beta$ (IL-1 $\beta$ ), 6 (IL-6) e fator de necrose tumoral $\alpha(\text { TNF- } \alpha)^{(2,3)}$.

A FOP é uma doença genética rara do tecido conjuntivo, caracterizada por ossificação disseminada em tecidos moles e alterações congênitas das extremidades. Seu diagnóstico baseia-se na história clínica, no exame físico e nos achados radiográficos, devendo-se evitar traumas e biópsias, pois podem acarretar o aparecimento de novas ossificações ${ }^{(15)}$. Um diagnóstico inicial incorreto dessa patologia, como no caso 2, ocasiona estresse desnecessário nos familiares e pacientes.

O tratamento da calcinose ainda não está bem estabelecido. Essa dificuldade na sua abordagem ocorre pela sua raridade e pela história natural variável e pouco previsível. No entanto, existem relatos na literatura do uso de várias medicações, com ou sem sucesso, no tratamento da calcinose ${ }^{(7-11,16-18)}$.

O mecanismo de ação dos bisfosfonatos na regressão da calcinose não é bem estabelecido. Os bisfosfonatos inibem a reabsorção óssea, reduzem o turn over do cálcio, causam destruição dos macrófagos e inibem a produção de citocinas inflamatórias, como as IL-1 $\beta$, IL-6 e TNF- $\alpha$, revertendo parcialmente a progressão das calcificações por inibir posteriores deposições de cálcio ${ }^{(2)}$. A inibição da reabsorção óssea diminui o cálcio disponível, reduzindo seu depósito nas calcificações. Os bisfosfonatos não removem tecidos calcificados previamente depositados, e o mecanismo de melhora da calcinose já estabelecida é desconhecido. Pode ser que o alendronato ligue-se ao cálcio e seja retirado pelos macrófagos. O efeito dos bisfosfonatos nos macrófagos pode explicar a redução da inflamação nas áreas calcificadas ${ }^{(3)}$. Seu efeito benéfico não é aplicado a todos os bisfosfonatos, já que existem estudos demonstrando falta de eficácia com o uso de etidronato ${ }^{(19}$, ${ }^{20)}$ e melhora das calcificações com o uso do clodronato ${ }^{(21)}$. 
Essas respostas diversas podem ser explicadas pelas diferentes características biológicas dessas medicações ${ }^{(22)}$.

Mukamel et al ${ }^{(3)}$ observaram uma rápida melhora das calcificações distróficas com alendronato $(10 \mathrm{mg} / \mathrm{dia}) \mathrm{em}$ um paciente de 6 anos com DMJ que antes havia utilizado diltiazen sem sucesso. Um ano após o início do tratamento, o paciente apresentou resolução quase completa das calcificações.

Ambler et al ${ }^{(11)}$ relataram um paciente com DMJ e calcinose subcutânea que não respondeu ao probenecid ( $1 \mathrm{~g} /$ dia $)$ e diltiazen $(30 \mathrm{mg} / \mathrm{dia})$, tendo apresentado melhora das calcificações um mês após o início do tratamento com alendronato $(10 \mathrm{mg} / \mathrm{dia})$.

Em crianças, a experiência com o alendronato e outros bisfosfonatos é muito limitada, e seu uso é reservado para pacientes com osteoporose idiopática juvenil, osteoporose induzida por glicocorticóides, osteogênese imperfeita, FOP, calcificação arterial infantil e displasia fibrosa ${ }^{(23-25)}$.

O diltiazen, um bloqueador do canal de cálcio, inibe o influxo de cálcio para as células, o crescimento e a proliferação do tecido muscular liso dos vasos e dos fibroblastos. Também inibe a síntese da matriz protéica extracelular, entretanto, o provável mecanismo de ação na calcinose seria pela diminuição da concentração de cálcio intracelular muscular, reduzindo a formação de cristais ${ }^{(17)}$.

Existem poucos relatos na literatura sobre o uso do diltiazen em pacientes com DMJ e calcinose. Oliveri et al ${ }^{(26)}$ descreveram uma paciente de 8 anos com DMJ que desenvolveu calcificações distróficas 26 meses após o diagnóstico. As calcificações tornaram-se generalizadas, apesar do tratamento da miopatia com corticosteróide e medicações imunossupressoras. Os autores utilizaram diltiazen $(5 \mathrm{mg} / \mathrm{kg} / \mathrm{dia})$, pamidronato oral $(4 \mathrm{mg} / \mathrm{kg} / \mathrm{dia})$, cálcio e vitamina $\mathrm{D}$. Após 21 meses de tratamento, observou-se regressão clínica e radiológica da calcinose. Embora o diltiazen tenha sido associado à regressão da calcinose, devemos considerar que o paciente também fazia uso de pamidronato. Também nesse caso, a calcinose ocorreu após o controle da atividade da doença.

A IGEV é um bloqueador do receptor Fc dos capilares endoteliais e inibidor da ativação do complemento, prevenindo o dano mediado pelo complemento aos vasos sangüíneos $\mathrm{e}$ às fibras musculares ${ }^{(27)}$. Ela tem sido utilizada para tratar manifestações relacionadas ao sistema imune e doenças inflamatórias, como a dermatomiosite. No entanto, seu uso no tratamento da calcinose ainda não está bem estabelecido. Existem relatos na literatura do uso de IGEV no tratamento da DMJ resistente ao corticosteróide e a outros imunossu- pressores (metotrexate, ciclosporina, azatioprina), algumas vezes demonstrando também melhora da calcinose ${ }^{(28-32)}$.

Existem na literatura relatos de casos do uso de algumas medicações como a hidroxicloroquina, a ciclosporina, a colchicina e o infliximabe com o intuito de controlar a atividade inflamatória da DMJ. No entanto, nesses trabalhos, os autores observaram também regressão importante ou retardo na progressão da calcinose após tratamento por dois a dez meses com essas medicações. A melhora da calcinose pode ter ocorrido pelo melhor controle da doença de base ${ }^{(33-37)}$.

A colchicina previne ou reduz a inflamação secundária aos depósitos de cálcio, entretanto, não têm sido observados redução ou desaparecimento dos depósitos de cálcio com essa medicação ${ }^{(35,36)}$.

Algumas medicações como o anti-TNF $\alpha$, o tacrolimus, o micofenolato mofetil e o leflunomide ainda necessitam de estudos para avaliar sua eficácia no tratamento da DMJ com calcinose secundária. Maillard et al ${ }^{(37)}$ observaram retardo na progressão e regressão parcial da calcinose em dois pacientes com DMJ persistente após dois a dez meses de tratamento com infliximabe (anti-TNF $\alpha$ ) na dose de 3 a $5 \mathrm{mg} / \mathrm{kg} /$ por aplicação.

A injeção intralesional de corticosteróide pode ser eficaz na regressão da calcinose pela redução do processo inflamatório local ${ }^{(38)}$.

Em alguns pacientes com calcinose volumosa, com dor crônica importante, com perda de função articular, com infecções recorrentes, com drenagem de secreções e com ulcerações, a cirurgia pode estar indicada ${ }^{(18)}$. Todos os pacientes devem ser orientados a evitar traumas.

Em resumo, não existe um tratamento específico e eficaz para a calcinose. A experiência com poucos casos sugere que o diltiazen parece ser uma medicação benéfica no tratamento da calcinose em alguns pacientes com DMJ refratária a outras terapias. Em nosso paciente do caso 1, a melhora provavelmente se deve à introdução do diltiazen.

Em relação ao paciente do caso 2, a importante melhora da calcinose com a IGEV, provavelmente se deve a um melhor controle da atividade inflamatória da DMJ.

Nossos relatos, em conjunto com outros da literatura, sugerem um benefício da terapia com diltiazen ou IGEV, na calcinose associada à DMJ. No entanto, não há estudos controlados e randomizados com essas terapias no tratamento dessa complicação.

A calcinose está geralmente relacionada a um retardo no diagnóstico e tratamento inadequado da DMJ. Essa complica- 
ção ocorre na vigência de atividade da doença. É importante ter em mente que a ausência do quadro de fraqueza muscular e/ou de aumento de enzimas musculares pode não ser suficiente para afastar atividade e a calcinose pode ser a única expressão desta. Seu aparecimento pode ser evitado com o uso precoce de imunossupressores em pacientes com atividade persistente de doença. Tão importante quanto tratar a calcinose já existente é prevenir o aparecimento de novas calcificações ${ }^{(39)}$.

\section{Declaramos a inexistência de conflitos de interesse.}

\section{REFERÊNCIAS}

1. Ramanan AV, Feldman BM: Clinical features and outcomes of juvenile dermatomyositis and other childhood onset myositis syndromes. Rheum Dis Clin North Am 28: 833-57, 2002.

2. Boulman N, Slobodin G, Rozenbaum M, Rosner I: Calcinosis in rheumatic diseases. Semin Arthritis Rheum 34: 805-12, 2005.

3. Mukamel M, Horev G, Mimouni M: New insight into calcinosis of juvenile dermatomyositis: a study of composition and treatment. J Pediatr 138: 763-6, 2001.

4. Ansell BM: Juvenile dermatomyositis. Rheum Dis Clin North Am 17: 931-42, 1991.

5. Fisler RE, Liang MG, Fuhlbrigge RC, Yalcindag A, Sundel RP: Aggressive management of juvenile dermatomyositis results in improved outcome and decreased incidence of calcinosis. J Am Acad Dermatol 47: 505-11, 2002.

6. Bowyer SL, Blane CE, Sullivan DB, Cassidy JT: Childhood dermatomyositis: Factors predicting functional outcome and development of dystrophic calcification. J Pediatr 103: 882-8, 1983.

7. Martinez-Cordero E, Lopez-Zepeda J, Choza-Romero F: Calcinosis in childhood dermatomyositis (letter). Clin Exp Rheumatol 8: 198-200, 1990.

8. Berger RG, Featherstone GL, Raasch RH, McCartney WH, Hadler NM: Treatment of calcinosis universalis with low-dose warfarin. Am J Med 83: 72-6, 1987.

9. Aihara J, Mori M, Ibe M, et al: A case of juvenile dermatomyositis with calcinosis universalis. Remarkable improvement with aluminum hydroxide therapy. Ryumachi 34: 879-84, 1994.

10. Dent CE, Stamp TCB: Treatment of calcinosis circumscripta with probenecid. BMJ 1: 216-8, 1972.

11. Ambler GR, Chaitow J, Rogers M, McDonald DW, Ouvrier RA: Rapid improvement of calcinosis in juvenile dermatomyositis with alendronate therapy. J Rheumatol 32: 1837-9, 2005.

12. Romero KT, Terreri MT, Len CA, Hilário MO: Dermatomiosite e polimiosite juvenis: Diagnóstico e tratamento. Rev Paul Pediatria 21: 223-7, 2003.

13. Wananukul S, Pongprasit P, Wattanakrai P: Calcinosis cutis presenting years before other clinical manifestations of juvenile dermatomyositis: Report of two cases. Aust J Dermatol 58: 202-5, 1997.

14. Sogabe T, Silva CAA, Kiss MH: Clinical and laboratorial characteristics of 50 children with dermato/polymyositis. Rev Bras Reumatol 36: 351-8, 1996.

15. Buyse G, Silberstein J, Goemans N, Casaer P: Fibrodysplasia ossificans progressiva: still turning into wood after 300 years? Eur J Pediatr 154: 694-9, 1995.
16. Miyamae T, Mori M, Inamo Y, et al: Multi-center analysis of calcinosis in children with juvenile dermatomyositis. Ryumachi 43: 538-43, 2003

17. Ichiki Y, Akiyama T, Shimozawa N, Suzuki Y, Kondo N, Kitajima $\mathrm{Y}$ : An extremely severe case of cutaneous calcinosis with juvenile dermatomyositis, and successful treatment with diltiazem. Br J Dermatol 144: 894-7, 2001.

18. Liniger P, Slongo T, Eckhardt O: Tumoral calcinosis and atypical juvenile dermatomyositis: case report. Eur J Pediatr Surg 8: 382$4,1998$.

19. Murphy E, Freaney R, Bresnihan B, McKenna M, Fitzgerald O: Evidence for increased bone turnover in patients with dystrophic calcification. Arthritis Rheum 39: S97, 1996.

20. Metzger AL, Singer FR, Bluestone R, Pearson CM: Failure of disodium etidronate in calcinosis due to dermatomyositis and scleroderma. N Engl J Med 291: 1294-6, 1974.

21. Castro GRW, Sampaio-Barros PD, Appenzeller S, Marques Neto JF, Sâmara AM: Uso do clodronato endovenoso na calcinose difusa em uma criança com síndrome de superposição esclerose sistêmica e dermatomiosite. Rev Bras Reumatol 44: 90-3, 2004.

22. Fleish H: Bisphosphonates: mechanism of action. Endocr Rev 19: 80-100, 1998.

23. Bianchi M, Cimaz R, Bardare M, et al: Efficacy and safety of alendronate for the treatment of osteoporosis in diffuse conective tissue diseases in children: a prospective multicenter study. Arthritis Rheum 43: 1960-6, 2000.

24. Rudge S, Hailwood S, Horne A, Lucas J, Wu F, Cundy T: Effects of once-weekly oral alendronate on bone in children on glucocorticoid treatment. Rheumatology 44: 813-8, 2005.

25. Glorieux F, Bishop N, Plotkin H, Chabot G, Lanoue G, Travers $\mathrm{R}$ : Cyclic administration of pamidronate in children with severe osteogenesis imperfecta. N Engl J Med 339: 947-52, 1998.

26. Oliveri MB, Palermo R, Mautalen C, Hubscher O: Regression of calcinosis during diltiazen treatment in juvenile dermatomyositis. J Rheumatol 23: 2152-5, 1996.

27. Ruetter A, Luger TA: Efficacy and safety of intravenous immunoglobulin for immune-mediated skin disease. Am J Clin Dermatol 5: 153-60, 2004.

28. Al-Mayouf SM, Laxer RM, Schneider R, Silverman ED, Feldman BM: Intravenous immunoglobulin therapy for juvenile dermatomyositis: efficacy and safety. J Rheumatol 27: 2498-503, 2000.

29. Vedanarayanan V, Subramony SH, Ray LI, Evans OB: Treatment of childhood dermatomyositis with high dose intravenous immunoglobulin. Pediatr Neurol 13: 336-9, 1995. 
30. Cherin P, Piette JC, Wechsler B, et al: Intravenous gamma globulin as first line therapy in polymyositis and dermatomyositis: an open study in 11 adult patients. J Rheumatol 21: 1092-7, 1994.

31. Lang BA, Laxer RM, Murphy G, Silverman ED, Roifman CM: Treatment of dermatomyositis with intravenous gammaglobulin. Am J Med 91: 169-72, 1991.

32. Bodemer C, Teillac D, Le Bourgeois M, et al: Efficacy of intravenous immunoglobulins in sclerodermatomyositis. Br J Dermatol 123: 545-51, 1990.

33. Olson NY, Lindsley CB: Adjunctive use of hydroxychloroquine in childhood dermatomyositis. J Rheumatol 16: 1545-7, 1989.

34. Zeller V, Cohen P, Prieur AM, Guillevin L: Cyclosporin a therapy in refractory juvenile dermatomyositis. Experience and longterm followup of 6 cases. J Rheumatol 23: 1424-7, 1996.

35. Fuchs D, Fruchter L, Fishel B, Holtzman M, Yaron M: Colchicine suppression of local inflammation due to calcinosis in dermatomyositis and progressive systemic sclerosis. Clin Rheumatol 5: 527-30, 1986.
36. Vereecken P, Stallenberg B, Tas S, de Dobbeleer G, Heenen M: Ulcerated dystrophic calcinosis cutis secondary to localised linear scleroderma. Int J Clin Pract 52: 593-4, 1998.

37. Maillard SM, Wilkinson N, Riley P, Beresford M, Davidson J, Murray KJ: The treatment of persistent severe idiopathic inflammatory myositis with anti-TNFalpha therapy. Arthritis Rheum 46 (suppl): S 307, 2002.

38. Hazen PG, Walker AE, Carney JF, Stewart JJ: Cutaneous calcinosis of scleroderma: successful treatment with intralesional adrenal steroids. Arch Dermatol 118: 366-7, 1982.

39. Rider LG: Calcinosis in juvenile dermatomyositis: pathogenesis and current therapies. Pediatric Rheumatology Online Journal. <www.pedrheumoonlinejournal.org/april/calinosis. html.> Modified from Presentation, "Treatment of Refractory Juvenile Dermatomyositis", 66th Annual American College of Rheumatology Meeting, October 2002, New Orleans. 